\title{
Variational Iteration Method Used to Solve a SIR Epidemic Model of Tuberculosis
}

\author{
Gabariela Purnama Ningsi, Sudi Mungkasi \\ \{ningsilatib5@gmail.com ${ }^{1}$, sudi@usd.ac.id² ${ }^{2}$. \\ Department of Mathematics Education, Universitas Sanata Dharma, Yogyakarta, Indonesia ${ }^{1}$, \\ Department of Mathematics, Universitas Sanata Dharma,Yogyakarta, Indonesia ${ }^{2}$
}

\begin{abstract}
This paper discusses the variational iteration method for solving non-linear differential equation systems. To be specific, the system we consider is the SIR (Susceptible-Infectious-Recovered) model of tuberculosis. Based on our research results, the calculation using the variational iteration method can be done quite easily. The method can predict the exact solution accurately at an arbitrary time.
\end{abstract}

Keywords: SIR model, tuberculosis, variational iteration method.

\section{Introduction}

Differential equations occur not only in mathematics, but actually also in problems of everyday life. They also occur in other fields of sciences, such as, economics, physics, biology, computers science, etc. [1]-[3]. A system of ordinary differential equations consists of some ordinary differential equations that fulfill the nature that an unknown function depends only on one independent variable [3].

One of problems in everyday life and in the field of biology that can be modeled into the form of non-linear differential equations is the problem of the spread of infectious diseases. To model this problem, we can use the SIR model. In the SIR model [4]-[5], the population studied will be grouped into three groups labeled $S, I$, and R. Here, $S(t)$ is a group of susceptible individuals (Susceptibles) against diseases that are not infected in time $t$; $I(t)$ is a group of infected individuals (Invectives) within $t$, this group of infections is able to transmit the disease to vulnerable groups (Susceptibles) in case of direct contact; $\mathrm{R}(\mathrm{t})$ is a group of individuals who have been infected and recovered from the disease, and will not be infected again (permanently recovered).

In this paper, we solve the SIR model for the spread of tuberculosis (TB) using a variational iteration method. Variational iteration method is a method used to solve linear and nonlinear differential equations that will predict solutions quickly, easily, effectively, and accurately [6][7].

This paper is written in the following structure. In Section 2, we will write about the SIR model for the spread of tuberculosis, which is the problem we want to solve. In Section 3, we will write about the variational iteration method to solve the SIR model. Results of mathematical computation will be presented in Section 4. Conclusions will be written in Section 5. 


\section{Mathematical Model}

Side [8] has modeled a non-linear differential equation system for the spread of tuberculosis (TB) by using the SIR model as follows:

$$
\begin{gathered}
\frac{d x}{d t}=\mu_{h}-\beta_{h} x-\gamma \beta_{h} x y-\mu_{h} x \\
\frac{d y}{d t}=\beta_{h} x-\alpha y \\
\frac{d z}{d t}=\gamma \beta_{h} x y-\eta z
\end{gathered}
$$

where, $\quad x(t)=\frac{S_{h}}{N_{h}}, y(t)=\frac{I_{h}}{N_{h}}, z(t)=\frac{I_{i}}{N_{h}}, \alpha=\mu_{h}+\delta_{h}, \eta=\mu_{h}+\varphi_{h}$.

Here:

$N_{h}$ is the total population at a certain time,

$S_{h}$ is the number of people suspected of being susceptible to tuberculosis at any given time,

$I_{h}$ is the number of people infected with TB at a certain time,

$R_{h}$ is the number of people who recover from tuberculosis at a certain time,

$\varphi_{h} I_{i}$ is the number of people recovering from an epidemic,

$\mu_{h}$ is the rate of birth or death in the human population,

$\beta_{h}$ is the level of suspected human being infected,

$\gamma$ is the estimated level of population vulnerable to infection,

$\delta_{h}$ is a parameter of the number of infected populations that are cured, and

$\varphi_{h}$ is a parameter of the number of infected populations who are cured.

\section{Variational Iteration Method}

Variational iteration method (VIM) was introduced by He [9]. VIM is the result of modification of the general Lagrange multiplier method which has been proven to find a solution easily from differential equations [8]-[10]. In VIM, there are 3 main concepts used to solve a differential equation, the Lagrange multiplier, the correction function and the restricted variation [3]. To solve the system (1) of differential equations (1a)-(1c) above, we will follow the writings of Rangkuti, et al. [11].

To illustrate the procedure in this variational iteration method, we consider the following differential equation:

$$
L \mu_{i}(t)+N \mu_{i}(t)=g_{i}(t), \quad i=1,2,3, \ldots, n
$$

where $L$ is linear operator, $N$ is nonlinear operator, and $g_{i}(t)$ is nonhomogeneous term of the differential equation. According to the variational iteration method, the terms of the sequence $\mu_{i}(t)$ are made in such a way that this finds the right solution of the mathematical model $\mu_{i}(t)$ can be calculated with the following correction functions: 


$$
\mu_{i, n+1}(t)=\mu_{i, n}(t)+\int_{0}^{t} \lambda_{i}\left[L \mu_{i, n}(s)+N \mu_{i, n}(s)-g_{i}(t)\right] d s
$$

where, $\lambda_{i}, i=1,2,3, \ldots, n$ is a Lagrange multiplier that can be identified optimally through variational theory, index $n$ shows the $n$-th order approximation, $\tilde{\mu}_{i, n}(s)$ is restricted variation, that is, $\delta \tilde{\mu}_{i, n}(s)=0[11]$.

The correction functional of the differential equation system [1] will be obtained as follows:

$$
\begin{aligned}
& x(n+1)=x_{n}+\int_{0}^{t} \lambda_{1}\left[\frac{d x_{n}}{d s}-\mu_{h}+\beta_{h} x_{n}+\gamma \beta_{h} \tilde{x}_{n} \tilde{y}_{n}+\mu_{h} x_{n}\right] d s \\
& y(n+1)=y_{n}+\int_{0}^{t} \lambda_{2}\left[\frac{d y_{n}}{d s}-\beta_{h} \tilde{x}_{n}+\alpha y_{n}\right] d s \\
& z(n+1)=z_{n}+\int_{0}^{t} \lambda_{3}\left[\frac{d z_{n}}{d s}-\gamma \beta_{h} \tilde{x}_{n} \tilde{y}_{n}+\eta z_{n}\right] d s
\end{aligned}
$$

where $\tilde{x}_{n}, \tilde{y}_{n}$, dan $\tilde{z}_{n}$ are restricted variations, that is, $\delta \tilde{x}_{n}=0, \delta \tilde{y}_{n}=0$, dan $\delta \tilde{z}_{n}=0$. From equations (4-6) we will get the following equations:

$$
\begin{gathered}
\delta x(n+1)=\delta x_{n}+\delta \int_{0}^{t} \lambda_{1}\left[\frac{d x_{n}}{d s}-\mu_{h}+\beta_{h} x_{n}+\gamma \beta_{h} \tilde{x}_{n} \tilde{y}_{n}+\mu_{h} x_{n}\right] d s, \\
\delta x(n+1)=\delta x_{n}+\delta \int_{0}^{t} \lambda_{1}\left[\frac{d x_{n}}{d s}-\mu_{h}+\beta_{h} x_{n}+\mu_{h} x_{n}\right] d s ; \\
\delta y(n+1)=\delta y_{n}+\delta \int_{0}^{t} \lambda_{2}\left[\frac{d y_{n}}{d s}-\beta_{h} \tilde{x}_{n}+\alpha y_{n}\right] d s, \\
\delta y(n+1)=\delta y_{n}+\delta \int_{0}^{t} \lambda_{2}\left[\frac{d y_{n}}{d s}+\alpha y_{n}\right] d s ; \\
\delta z(n+1)=\delta z_{n}+\delta \int_{0}^{t} \lambda_{3}\left[\frac{d z_{n}}{d s}-\gamma \beta_{h} \tilde{x}_{n} \tilde{y}_{n}+\eta z_{n}\right] d s, \\
\delta z(n+1)=\delta z_{n}+\delta \int_{0}^{t} \lambda_{3}\left[\frac{d z_{n}}{d s}+\eta z_{n}\right] d s .
\end{gathered}
$$

By using integration by parts, equations (7-9) will be:

$$
\begin{gathered}
\delta x(n+1)=\delta x_{n}+\delta \lambda_{1} x_{n}-\int_{0}^{t}\left[x_{n} \delta \lambda_{1}^{\prime}-\delta \lambda_{1}\left(\beta_{h}+\mu_{h}\right) x_{n}\right] d s, \\
\delta x(n+1)=\delta x_{n}\left(1+\lambda_{1}\right)-\int_{0}^{t}\left[\left(\lambda_{1}^{\prime}-\left(\beta_{h}+\mu_{h}\right) \lambda_{1}\right) x_{n} \delta\right] d s
\end{gathered}
$$




$$
\begin{gathered}
\delta y(n+1)=\delta \quad y_{n}+\delta \lambda_{2} y_{n}-\int_{0}^{t}\left[\delta \lambda_{2} y_{n}\right] d s+\int_{0}^{t}\left[\alpha \delta \lambda_{2} y_{n}\right] d s, \\
\left.\delta y(n+1)=\delta y_{n}\left(1+\lambda_{2}\right)-\int_{0}^{t}\left[\lambda_{2}^{\prime}-\lambda_{2} \alpha\right) y_{n} \delta\right] d s ; \\
\delta z(n+1)=\delta z_{n}+\delta \lambda_{3} z_{n}-\int_{0}^{t}\left[\delta \lambda_{3}^{\prime} z_{n}\right] d s+\int_{0}^{t}\left[\delta \eta y_{n}\right] d s, \\
\delta z(n+1)=\delta z_{n}\left(1+\lambda_{3}\right)-\int_{0}^{t}\left[\left(\lambda_{3}^{\prime}-\eta\right) z_{n} \delta\right] d s .
\end{gathered}
$$

Therefore, for each variations $\delta x_{n}, \delta y_{n}, \delta z_{n}$ and $\delta x_{n}^{\prime}, \delta y_{n}^{\prime}, \delta z_{n}^{\prime}$ we obtain stationary conditions as follows:

$$
\begin{array}{cc}
\delta x_{n}:\left(1+\left.\lambda_{1}(t)\right|_{s=t}=0\right. & \delta x_{n}:\left.\left(\lambda_{1}^{\prime}-\left(\beta_{h}+\mu_{h}\right) \lambda_{1}\right)\right|_{s=t}=0 \\
\delta y_{n}:\left(1+\left.\lambda_{2}(t)\right|_{s=t}=0\right. & \delta y_{n}:\left.\left(\lambda_{2}{ }^{\prime}-\lambda_{2} \alpha\right)\right|_{s=t}=0 \\
\delta z_{n}:\left(1+\left.\lambda_{3}(t)\right|_{s=t}=0\right. & \delta z_{n}:\left.\left(\lambda_{3}{ }^{\prime}-\eta\right)\right|_{s=t}=0
\end{array}
$$

The solution of equation (13) above is as follows:

$$
\begin{aligned}
& \lambda_{1}(t)=-1 \\
& \lambda_{2}(t)=-1 \\
& \lambda_{3}(t)=-1
\end{aligned}
$$

$$
\begin{gathered}
\lambda_{1}(s)=-e^{\left(\beta_{h}+\mu_{h}\right)(s-t)} \\
\lambda_{2}(s)=-e^{\alpha(s-t)} \\
\lambda_{3}(s)=-1+\eta(s-t)
\end{gathered}
$$

Then we obtain:

$$
\begin{aligned}
& \lambda_{1}(s)=-1 \\
& \lambda_{2}(s)=-1 \\
& \lambda_{3}(s)=-1
\end{aligned}
$$

Substitution of the Lagrange multipliers (15) to the initial equations (4)-(6) so that it is obtained:

$$
\begin{gathered}
x(n+1)=x_{n}-\int_{0}^{t}\left[\frac{d x_{n}}{d s}-\mu_{h}+\beta_{h} x_{n}+\gamma \beta_{h} x_{n} y_{n}+\mu_{h} x_{n}\right] d s \\
y(n+1)=y_{n}-\int_{0}^{t}\left[\frac{d y_{n}}{d s}-\beta_{h} x_{n}+\alpha y_{n}\right] d s \\
z(n+1)=z_{n}-\int_{0}^{t}\left[\frac{d z_{n}}{d s}-\gamma \beta_{h} x_{n} y_{n}+\eta z_{n}\right] d s
\end{gathered}
$$

Equations (16)-(18) will provide the estimate solution to the spread of TB disease. 


\section{Results and Discussion}

To do the iteration, we need initial data. Suppose that the initial data is $x_{0}=\frac{8377828}{8386763}=0.998935, y_{0}=\frac{8000}{8386763}=0.000954$, $z_{0}=\frac{939}{838676}=0.000112, \mu_{h}=0.000046, \beta_{h}=0.326666, \varphi_{h}=0.003700$ [8].

Then the variational iteration solutions of equations (16)-(18) are as follows:

$$
\begin{gathered}
x_{1}=0.998935-0.3263563769 t \\
y_{1}=0.00094+0.326278723 t \\
z_{1}=0.000112+0.00003790582171 t \\
x_{2}=0.9989935-0.6527127538 t-0.0244394405 t^{2}+0.004282326039 t^{3} \\
y_{2}=0.000954+0.6525574468 t-0.1200770128 t^{2} \\
z_{2}=0.000112+0.00007581147385 t+0.0130949876 t^{2}-0.004282326039 t^{3} \\
x_{3}=0.998935-0.9790691307 t+0.280588245 t^{2}-0.00432526614 t^{3} \\
-0.007005728528 t^{4}+0.0003392729947 t^{5}+0.00002067942587 t^{6} \\
y_{3}=0.000954+0.9788361702 t-0.3602310384 t^{2}+0.03550917866 t^{3} \\
+0.001398890318 t^{4} \\
z_{4}=0.000954+1.305114894 t-0.7204620768 t^{2}+0.1420367147 t^{3}-0.001479703929 t^{4} \\
0.03237662782 t^{4}-0.005937713462 t^{5}+0.0001130954128 t^{6}+ \\
0.0001245346764 t^{7}-0.0000143487926 t^{8}-2.092161586[10]^{-7} t^{9}+ \\
4.861773107[10]^{-8} t^{10}+1.163378447[10]^{-9} t^{11} \\
+0.005622682816 t^{4}-0.0003392729947 t^{5}-0.00002067942587 t^{6} \\
+0.008205506472 t^{5}-0.0002226690546 t^{6}-0.0001312134279 t^{7}+0.0000143487926 t^{8} \\
z_{4}=0.998935-1.305425507 t+0.56117649 t^{2}-0.04299502079 t^{3}-0.03224810867 t^{4} \\
+0.9
\end{gathered}
$$




$$
\begin{aligned}
& x_{5}=0.998935-1.631781884 t+0.9352941499 t^{2}-0.1288991823 t^{3}-0.0911832581 t^{4} \\
& +0.04477506009 t^{5}-0.00564592224 t^{6}-0.00127213563 t^{7}+0.0005468029644 t^{8}- \\
& 0.00005903753127 t^{9}-0.000005915359051 t^{10}+0.000002085411441 t^{11}- \\
& 1.295993491[10]^{-7} t^{12}-1.530653689[10]^{-8} t^{13}+2.255697091[10]^{-9} t^{14}- \\
& 4.813819307[10]^{-12} t^{15}-9.487319505[10]^{-12} t^{16}+5.00824738[10]^{-14} t^{17} \\
& +1.839325762[10]^{-14} t^{18}+3.160547325[10]^{-16} t^{19} \\
& y_{5}=0.000954+1.631392663 t-1.202075243 t^{2}+0.3558122488 t^{3}- \\
& 0.02152945954 t^{4}-0.01281807862 t^{5}+0.002890480005 t^{6}- \\
& 0.00007066854884 t^{7}-0.00004314855124 t^{8}+0.000004687262683 t^{9} \\
& +6.834380567[10]^{-8} t^{10}-1.588175974[10]^{-8} t^{11}-3.800361838[10]^{-10} t^{12} \\
& z_{5}=0.000112+0.0001895282606 t+0.1309498761 t^{2}-0.1771631783 t^{3}+ \\
& 0.105656311 t^{4}-0.03209270585 t^{5}+0.002877753842 t^{6}+0.001337781875 t^{7} \\
& -0.0005044004698 t^{8}+0.000054400335912 t^{9}+0.000005847789345 t^{10}- \\
& 0.000002069709567 t^{11}+1.299750808[10]^{-7} t^{12}+1.530652689[10]^{-8} t^{13}- \\
& 2.255697091[10]^{-9} t^{14}+4.813819307[10]^{-12} t^{15}+9.487319505[10]^{-12} t^{16}- \\
& 5.00824738[10]^{-14} t^{17}-1.839325762[10]^{-14} t^{18}-3.160547325[10]^{-16} t^{19}
\end{aligned}
$$

Using the Maple software, a graph of solutions of equations (31)-(33) above can be obtained in a Cartesius plane so that we can see the solution behavior of the three human populations ( $\mathrm{S}$, $\mathrm{I}$, and R). Figure 1 is a graph of the solutions of the three human population groups for the spread of TB disease with the SIR model and solved using the variational iteration method at the fifth iteration.

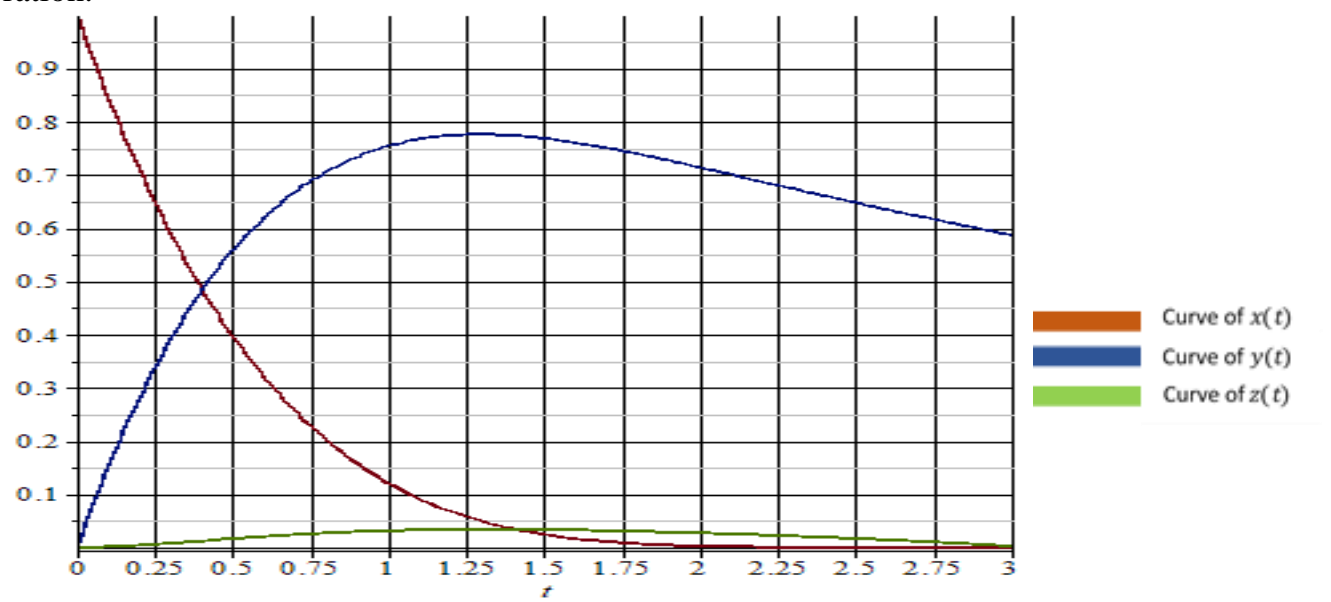

Fig. 1. Solutions to the SIR model obtained from the VIM at the fifth iteration

From Figure 1, we observe that: first, the more people infected with TB, the number of people who are vulnerable to the disease will also decrease until eventually all members of the population will be infected over time; second, the human population that is susceptible to disease 
is close to zero when 3 years have passed from the time when the disease spreads; third, the rapidly infected human population to the highest level reached $77.569 \%$ of the total human population. These are results obtained using the setting we use in this paper. Different results may occur if we have different setting. However, the phenomenon will be similar. Therefore, the variational iteration method has been able to solve the SIR model.

\section{Conclusion}

Based on our results, the variational iteration method can estimate the solution of a nonlinear differential equation system with an initial value. The system of nonlinear differential equations studied is a system of equations of the spread of tuberculosis in the SIR epidemic model. Variational iteration methods can approximate exact solutions at any given time and the approximate solutions are continuous at all time. In addition, the formulas contained in each iteration are not complicated. Therefore, calculations carried out using variational interation methods are easy.

Acknowledgments. The authors thank Universitas Sanata Dharma for the financial support.

\section{References}

[1] Haberman, R.: Mathematical Models: Mechanical Vibrations, Population Dynamics, and Traffic Flow. Englewood Cliffs: Prentice-Hall (1977)

[2] Li, M.Y., Shuai, Z.: Global-stability problem for coupled systems of differential equations on networks. Journal of Differential Equations, Vol. 248, pp. 1-20 (2010)

[3] Yuliyanto, B. D., Mungkasi, S.: Variational iteration method for solving the population dynamics model of two species. Journal of Physics: Conference Series, Vol. 795, pp. 012045 (2017)

[4] Brauer, F., Chavez, C.C.: Mathematical Models in Population Biology and Epidemiology. New York: Springer (2012)

[5] Murray, J. D:. Mathematical Biology: An Introduction. New York: Springer (2002)

[6] Shakeri, F., Dehghan, M.: Numerical solution of a biological population model using He's variational iteration method. Computers and Mathematics with Applications, Vol. 54, pp. 1197-1209 (2007).

[7] He, J.H., Wu, X. H.: Variational iteration method: new development and applications. Computers and Mathematics with Applications, Vol. 54, pp. 881-894 (2007)

[8] Side, S.: A susceptible-infected-recovered model and simulation for transmission of tuberculosis. Advanced Science Letters, Vol. 21, pp. 137-139 (2015)

[9] Salehpoor, E., Jafari, H.: Revised variational iteration method for solving systems of ordinary differential equations. Applications and Applied Mathematics, Special Issue No. 1, pp. 110-121 (2010) [10] Abbasbandy, S, Shivanian, E.: Application of the variational iteration method for system of nonlinear volterra's integro-differential equations. Mathematical and Computational Applications, Vol. 14, pp. 147-158 (2009)

[11] Rangkuti, Y. M., Side, S., Noorani, M. S.: Numerical analytic solution of sir model of dengue fever disease in South Sulawesi using homotopy perturbation method and variational iteration method. Journal of Mathematical and Fundamental Sciences, Vol. 46, pp. 91-105 (2014) 